\title{
Propuesta de un nuevo ajuste para el cálculo del rendimiento y análisis de su influencia en la estimación de la dosis glandular
}

\section{A new adjustment proposal for determining tube output and analysis of its influence on mean glandular dose estimation}

\author{
Noelia Solís Preciado ${ }^{1}$, José Ángel Merino Gestoso ${ }^{1}$, María Luisa Chapel Gómez ${ }^{2}$, Diego Jiménez Vegas ${ }^{1}$, \\ Breixo Carmelo Portas Ferradás ${ }^{1}$, Domingo Espinosa López ${ }^{1}$, Abel Niebla Piñero ${ }^{1}$ \\ 1 Hospital Universitario Nuestra Señora de Candelaria (Santa Cruz de Tenerife) \\ 2 Complejo Hospitalario de Toledo
}

Fecha de Recepción: 10/07/2019 - Fecha de Aceptación: 01/01/2020

En este trabajo se propone una nueva función de ajuste polinómica para la obtención del kerma en aire para cualquier kilovoltaje, frente al ajuste potencial recomendado por los protocolos europeo y español. Se ha medido el kerma y la capa hemirreductora en tres equipos de mamografía diferentes con cámara de ionización y detector semiconductor, para mamografía tanto en modo 2D como en tomosíntesis de mama. A partir de esos valores se ha estimado la dosis glandular siguiendo el modelo de Dance et al. Se ha obtenido una mejor adecuación entre el kerma medido y el calculado a la función propuesta que a la potencial. La estimación de la dosis glandular a partir del kerma obtenido mediante la función potencial de ajuste y la polinómica, muestran diferencias inferiores a un $2.5 \%$ en todos los equipos midiendo con la cámara de ionización, excepto para espesores de mama grandes (mayores de $6 \mathrm{~cm}$ ). Sin embargo, las diferencias obtenidas entre los detectores utilizados han resultado mayores, encontrándose en torno al 10\%. Por tanto, resulta fundamental conocer la respuesta de los diferentes detectores y el uso de una adecuada metodología de ajuste que minimice las incertidumbres obtenidas en la estimación de la dosis glandular.

Palabras clave: Dosis glandular, mamografía, tomosíntesis de mama, kerma, detectores en mamografía.

The aim of this work is to propose a new polynomial fitting function to obtain air kerma for any kilovoltage in comparison to exponential fitting recommended by European and Spanish protocols. Kerma and half-value layer have been measured on three different mammography systems with an ionization chamber and a semiconductor dosimeter, in both digital mammography and digital breast tomosynthesis. Glandular dose has been estimated from these values using the method by Dance et al. The function proposed reveals a better agreement than the exponential one between measured and calculated kerma. Glandular dose estimation calculated from kerma by both exponential and polynomial function shows differences of less than $2.5 \%$ for all mammography systems using the ionization chamber, except for big breasts (bigger than $6 \mathrm{~cm}$ ). Nevertheless, greater differences $(\sim 10 \%)$ are found between employed dosimeters. Therefore, knowing the dosimeters responses properly and using and accurate adjustment methodology is very important to minimize uncertainties in the glandular dose estimation.

Key words: Glandular dose, mammography, breast tomosynthesis, kerma, mammography dosimeters.

\footnotetext{
*Correspondencia: noeliasolispreciado@gmail.com https://doi.org/10.37004/sefm/2020.21.1.002
} 


\section{Introducción}

El cáncer de mama es el más frecuente en las mujeres en todo el mundo. Una herramienta clave para su detección es la mamografía (MD), por ello se realizan programas poblacionales de cribado mamográfico en los que se invita a mujeres sanas a realizarse exploraciones con rayos $X$. Sin embargo, la eficacia de la mamografía para detectar el cáncer de mama en estadios iniciales está acompañada de un bajo riesgo, pero no despreciable, de inducir cáncer por radiación en los tejidos fibroglandulares de la mama. ${ }^{1}$ Resulta por ello fundamental realizar una estimación de la dosis absorbida por la mama para asegurar que los niveles de dosis impartidos se encuentran dentro de los valores de referencia.

Para estimar el riesgo radiológico asociado a los exámenes mamográficos se utiliza la dosis glandular promedio (DG), recomendada por la Comisión Internacional de Unidades Radiológicas (ICRU) y la Comisión Internacional de Protección Radiológica (ICRP)., 2,3 La DG es una magnitud que no puede medirse directamente y se estima a partir de las medidas del kerma en aire sin retrodispersión en la superficie de entrada de la mama aplicando factores de corrección. Hay diferentes modelos para el cálculo de la DG basados en métodos de Montecarlo. ${ }^{4-8}$ La diferencia entre ellos se encuentra en los métodos de simulación, pero todos tienen en común la dependencia de los factores de conversión con el espesor de mama, su composición y las características del espectro de rayos $\mathrm{X}$ utilizado.

Por otra parte, la mamografía convencional presenta una limitación importante como es la acumulación de toda la información del volumen de la mama en un plano, provocando la superposición de estructuras y pudiendo dar lugar al enmascarado de lesiones o a su simulación.

El desarrollo de detectores digitales para mamografía en los últimos años ha impulsado la aparición de nuevas modalidades de imagen de mama con rayos $X$ como es la tomosíntesis (TDM), la cual se está implantando rápidamente en el ámbito clínico, existiendo numerosos estudios que evidencian su capacidad para mejorar el diagnóstico precoz del cáncer de mama.9-12

Durante la exploración de tomosíntesis el tubo de rayos $X$ se mueve describiendo un arco alrededor de la mama comprimida, generándose a partir de las distintas proyecciones un conjunto de cortes o planos paralelos al soporte de la mama. Cada una de estas proyecciones hay que considerarlas en la estimación de la DG para esta modalidad. La metodología para el cálculo de la dosis de radiación para un ángulo concreto se basa en multiplicar la DG estimada por un factor, que tiene en cuenta el mayor depósito de ener- gía que se origina al aumentar el ángulo de incidencia del haz de radiación como consecuencia del mayor recorrido de los fotones dentro de la mama. De acuerdo con ello, diferentes autores estimaron este nuevo factor siguiendo métodos de Montecarlo, ${ }^{13-15}$ el cual depende del ángulo de incidencia de cada proyección TDM y del espesor de la mama.

De acuerdo con lo anterior, para realizar la estimación de la dosis glandular (tanto en MD como en TDM) es fundamental realizar una buena caracterización dosimétrica de los equipos de mamografía. Para ello resulta imprescindible conocer muy bien la respuesta de los sistemas de detección que se utilicen en las medidas dosimétricas de los mamógrafos, tanto para el kerma como para la capa hemirreductora (CHR). Los instrumentos comúnmente utilizados son detectores de estado sólido y/o cámaras de ionización. Estas últimas presentan una respuesta prácticamente plana con la energía en el rango utilizado en mamografía; no así los semiconductores, cuya respuesta es dependiente de la calidad de haz, por lo que hay que disponer de curvas de calibración apropiadas para todas las calidades utilizadas en la práctica clínica. ${ }^{16}$

Avramova et al, ${ }^{17}$ en un estudio realizado sobre la influencia del procedimiento de medida en la DG, concluyeron que la medida del rendimiento del equipo y de la CHR está altamente influenciada por la configuración de medida, indicando la necesidad de estandarizar las mismas.

El objetivo de este trabajo es proponer una nueva metodología de ajuste para la obtención del kerma en aire para cualquier kVp y estudiar su influencia en la estimación de la DG.

\section{Material y métodos}

Las medidas de kerma y CHR se efectuaron en tres equipos de mamografía. Se realizaron en modo 2D en un equipo Senographe Essential de General Electric que dispone de doble pista anódica Molibdeno (Mo) y Rodio (Rh) y filtros de molibdeno (Mo) y rodio $(\mathrm{Rh})$, dando lugar a tres posibles combinaciones de ánodo/filtro: Mo/0.03 mm Mo, Mo/0.025 mm Rh y $\mathrm{Rh} / 0.025 \mathrm{~mm} \mathrm{Rh}$; en un Selenia Dimensions de Hologic con ánodo de tungsteno (W) y filtros de plata $(\mathrm{Ag})$ y rodio $(\mathrm{Rh})$ teniendo así las combinaciones W/0.05 mm Ag y W/0.05 mm Rh; y en un Mammomat Inspiration de Siemens con ánodo/filtro W/0.05 mm Rh.

Las medidas en el modo TDM se efectuaron en el mamógrafo Mammomat Inspiration que trabaja con calidad de haz W/0.05 mm Rh y el Selenia Dimensions que dispone de una calidad de haz de W/0.7 mm Al.

Para cada mamógrafo y calidad de haz disponible se realizaron medidas de rendimientos y $\mathrm{CHR}$ con una 


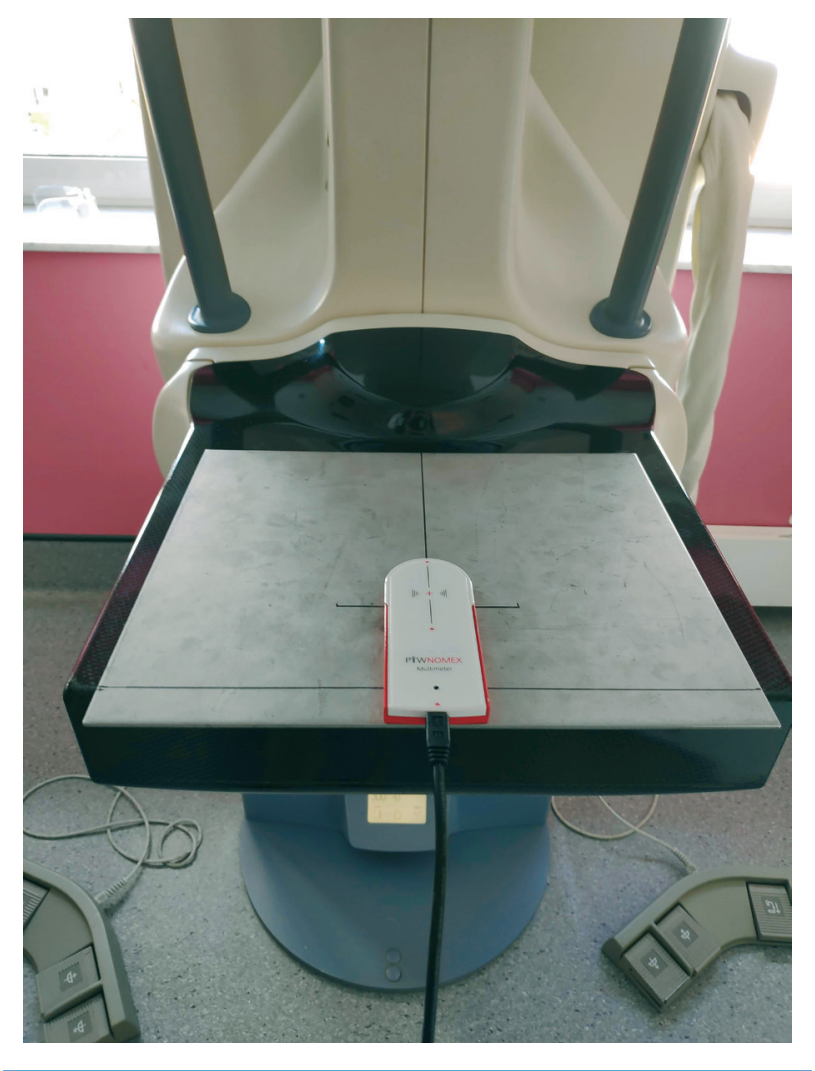

Fig. 1. Montaje del detector semiconductor NOMEX Multímetro (PTW).

cámara de ionización plano-paralela de volumen activo de $6 \mathrm{~cm}^{3}$ de PTW, y un detector semiconductor Nomex Multímetro, también de PTW. Para ambos detectores se empleó el Nomex Dosímetro, todo el conjunto con fecha de calibración de mayo de 2018 para todas las calidades de haz utilizadas en este trabajo, y con una desviación máxima en su respuesta de $\pm 0.5 \%$. Las medidas fueron realizadas entre el último trimestre de 2018 y el primero de 2019.

Se utilizó un rango de kVp hasta $35 \mathrm{kVp}$ para todas las calidades de haz de acuerdo con la máxima tensión que figura en los certificados de calibración de los dosímetros empleados.

Para la ejecución de las medidas se diseñó un método reproducible para permitir una reposición exacta de cada dosímetro en la unidad de mamografía, de manera que se centraron lateralmente y con su punto efectivo situado a $6 \mathrm{~cm}$ de la pared del tórax. Sobre el detector de imagen se colocó una lámina protectora. El dosímetro de estado sólido se dispuso directamente sobre la plancha de protección del soporte del mamógrafo (Fig. 1) y la cámara de ionización se situó a $10 \mathrm{~cm}$ sobre el soporte para evitar la radiación retrodispersa (Fig. 2). En principio, el kerma debería ser medido en contacto con la pala de compresión, pero como no todos los dosímetros presentan la misma

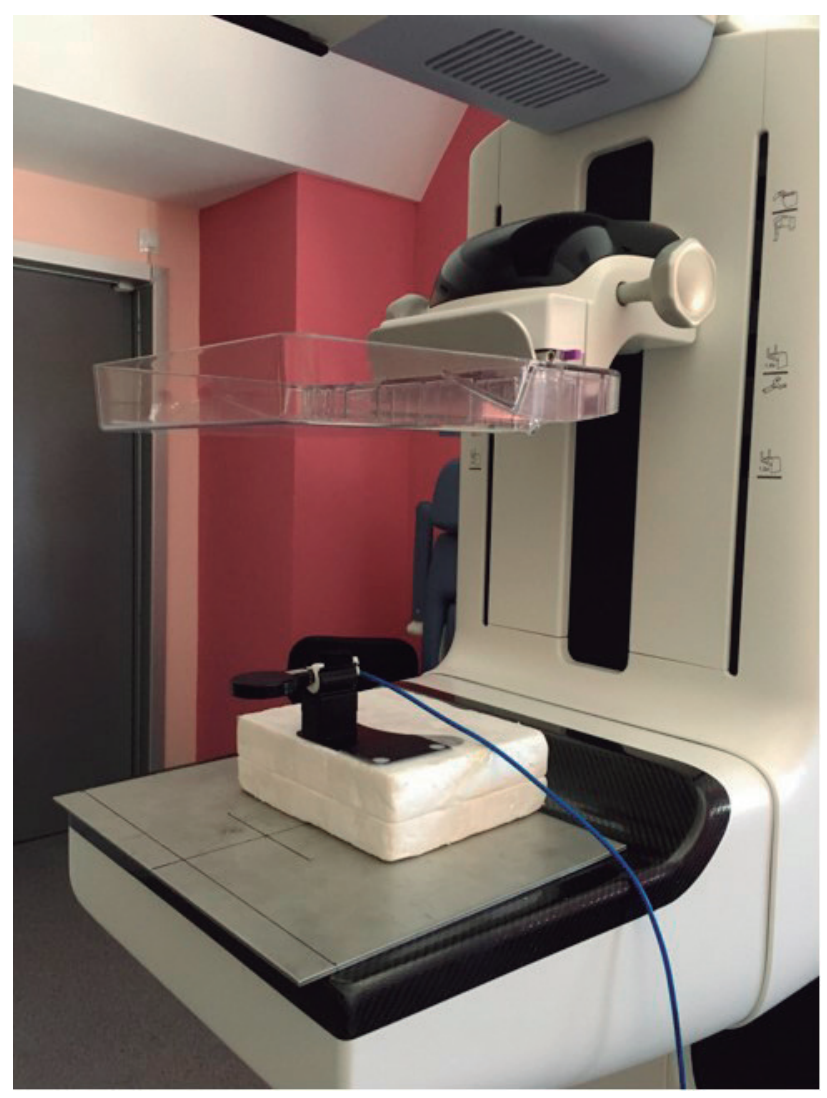

Fig. 2. Montaje de la cámara de ionización (PTW).

sensibilidad a la radiación dispersa, la pala de compresión se situó en la posición más alejada posible que nos permitía el equipo, con el fin de minimizar la contribución de radiación dispersa, como se indica en el Protocolo de Control de Calidad en Mamografía Digital de EFOM y el Protocolo Español de Calidad de Radiodiagnóstico, ${ }^{20,21}$ aplicando a las medidas un factor de 1.076 que tiene en cuenta la contribución de esta radiación.

\section{Realización de las medidas}

En modo 2D se tomaron medidas del kerma en aire (con errores del 3\% para la cámara de ionización y $1.5 \%$ para el multímetro) para distintos $\mathrm{kVp}$, para cada calidad de haz y para dos valores de mAs (50 mAs y $100 \mathrm{mAs}$ ), promediando los resultados de las medidas obtenidos. Se utilizó geometría de campo pequeño $(10 \times 10 \mathrm{~cm})$.

Inicialmente se midió con la cámara de ionización el kerma en el mamógrafo de Hologic para seis valores diferentes de kVp. Sin embargo, en los controles de calidad normalmente las medidas se realizan para cuatro valores de kilovoltaje. Por ello, se efectuaron los ajustes del kerma para los seis valores iniciales de kVp y 
se compararon los resultados con los ajustes realizados, tanto para la función polinómica como para la potencial, para solo cuatro puntos de kVp.

Para las medidas de la CHR con la cámara de ionización, se utilizaron filtros de Al de $99.9 \%$ de pureza y de $0.1 \mathrm{~mm}$ de espesor que se colocaron sobre la pala de compresión para cada calidad de haz, respetando siempre la misma disposición de los filtros en cada medida, para lo cual se numeraron los mismos adecuadamente. En el caso de NOMEX Multímetro no fue necesario el uso de los filtros de aluminio para determinar la $\mathrm{CHR}$, ya que estos equipos la proporcionan en cada exposición con un error asociado de un $2.8 \%$.

Las medidas en modo TDM se efectuaron en posición de $0^{\circ}$ y se colocó un colimador de plomo a la salida de la ventana. Las medidas se efectuaron a los mismos valores de $\mathrm{kVp}$ que en modo MD, a pesar de que en el rango clínico en modo tomosíntesis se alcanzan valores más altos de kilovoltaje, pero la calibración de los detectores en este estudio limitaba la tensión utilizada a un máximo de $35 \mathrm{kVp}$. Para las medidas de la CHR los filtros de Al se fijaron al colimador de plomo situado en la salida del tubo.

\section{Ajuste de los datos experimentales}

Puesto que tanto el rendimiento del equipo como la CHR dependen de la calidad de haz utilizada (ánodo/ filtro y $\mathrm{kVp}$ ), debería medirse ambos parámetros para cada espectro de rayos $X$ utilizado en la práctica clínica. Sin embargo, es posible reducir drásticamente las medidas utilizando la relación potencial que existe entre el kVp y el kerma $\left(K_{k V p}\right):{ }^{19}$

$$
K_{k V p}=A(k V p)^{n}
$$

Expresión que se convierte en lineal mediante transformaciones logarítmicas de acuerdo con la siguiente expresión:20,21

$$
\log \left(K_{k V p}\right)=\log A+n \cdot \log (k V p)
$$

Cada fabricante utiliza ajustes diferentes para el cálculo del kerma mostrado por sus equipos en cada exposición, y a partir del mismo estiman la dosis glandular. Algunos utilizan funciones potenciales, otros polinómicas e incluso otros mediante tablas de ajustes. Por ello en este estudio, además de ajustar los valores de rendimientos en función del $\mathrm{kVp}$ a una ecuación del tipo (2), se ajustó el kerma obtenido en las distintas medidas realizadas a una expresión polinómica del tipo:

$$
K_{k V p}=d+e \times k V p+f \times k V p^{2}
$$

Lo que permite obtener el valor de kerma para cualquier calidad de haz y kVp utilizado en la práctica clínica.

Para el cálculo de la capa hemirreductora (CHR) se utilizó el método de Robson, ${ }^{18}$ por lo que se midió la CHR para las distintas calidades de haz empleadas en la práctica clínica y varios puntos de kilovoltaje, y posteriormente se ajustó a una función polinómica del tipo:

$$
C H R=g+h \times k V p+j \times k V p^{2}
$$

Obteniendo así el valor de la CHR para cualquier kilovoltaje y calidad de haz.

Por tanto, y siguiendo la metodología propuesta, se efectuaron medidas de kerma en aire y de CHR para cuatro valores de $\mathrm{kVp}$.

Los ajustes se realizaron con el software Microsoft Excel.

\section{Dosis glandular}

Para la estimación de la dosis glandular se utilizó el método propuesto por las guías europeas que siguen el modelo de Dance y col. que convierte el kerma a la entrada de la mama o del maniquí en $\mathrm{DG}, 20$ usando tres factores según la siguiente expresión:

$$
D G=K \cdot g \cdot s \cdot c
$$

donde:

- $K$ es el kerma en aire (mGy) en la superficie de entrada de la mama.

- El factor $g$ (mGy/mGy) convierte el kerma en aire a dosis glandular asumiendo una glandularidad de la mama del 50\%. Su valor está tabulado en función del espesor de la mama comprimida y de la capa hemirreductora (CHR).

- El factor $c$ tiene en cuenta las diferentes glandularidades de la mama. Su valor depende del espesor, glandularidad de la mama y de la CHR.

- El factor $s$ considera las diferentes calidades de haz utilizadas. En el caso de una combinación ánodo/ filtro de W/Al, el factor s depende también del voltaje utilizado $(k \vee p)$.

El kerma en aire en la superficie de entrada de la mama se estima a partir de la técnica utilizada por cada mamógrafo para distintos espesores de PMMA con el control automático de exposición (espesor de mama comprimida, calidad del haz, kVp y mAs) mediante la expresión: 


$$
K=(m G y / m A s) \cdot m A s \cdot\left(f_{1} / f_{2}\right)^{2}
$$

donde:

- $m G y / m A s \circ Y(d)$, es el rendimiento del tubo medido en el punto de referencia con compresor y sin retrodispersión para la combinación ánodo/filtro y la tensión de trabajo.

- mAs es la carga del tubo utilizada para cada espesor de mama con control automático de exposición para obtener la imagen.

- $f_{1}$ es la distancia del centro del dosímetro al foco del tubo.

- $f_{2}$ es la distancia entre la superficie de entrada de la mama y el foco del tubo.

En el caso de TDM, para estimar la DG a partir del kerma en la superficie de entrada se utiliza la siguiente expresión:

$$
D G(T)=K \cdot g \cdot s \cdot c \cdot T
$$

donde $K$ es el kerma incidente en la superficie de entrada de la mama medido con el tubo fijo en la posición de $0^{\circ}$, utilizando el valor total de la carga $(m A s)$ de un barrido completo.

Cuando todas las proyecciones de tomosíntesis se adquieren con los mismos $m A s$, el factor $T$ se define como:

$$
T=\frac{1}{N} \sum_{i=0}^{N} t\left(\theta_{i}\right)
$$

siendo $t\left(\theta_{i}\right)$ el factor de tomosíntesis cuyo valor está tabulado para distintos ángulos $\theta_{i}$ y espesores de mama bajo compresión, y $N$ es el número de proyecciones. Los valores del factor $T$ se han tabulado para una serie de sistemas de tomosíntesis comercializados. ${ }^{13,22}$

\section{Resultados}

Los resultados de las medidas del kerma para 6 y 4 valores se muestran en la Tabla 1. Tal como se puede observar, las diferencias entre los valores medidos y los calculados con ambos ajustes resultan siempre menores para el caso de seis puntos, siendo inferiores además para el ajuste polinómico que para el potencial.

Como el objetivo final del cálculo del kerma es la estimación de la dosis glandular, se analizaron las discrepancias en los valores obtenidos de la misma a partir de los datos de ambos ajustes, encontrándose en el modo TDM diferencias menores del $1 \%$ y en el modo MD una diferencia media alrededor del 1.4\% para el ajuste polinómico y del 3.6\% para el ajuste potencial (Tabla 2).

Por tanto, y puesto que las variaciones encontradas no resultaron relevantes, el resto del estudio se realizó utilizando cuatro valores de las medidas del kerma para todos los mámografos y ambos detectores.

\section{Kerma en aire}

En la Tabla 3 se muestran las diferencias obtenidas para cada mamógrafo y calidad de haz en ambos detectores.

Las diferencias entre los datos medidos y los calculados resultan en todos los casos inferiores al $1 \%$ para

\begin{tabular}{|c|c|c|c|c|c|c|c|c|}
\hline \multirow{3}{*}{$\begin{array}{c}\text { Ánodo/ } \\
\text { Filtro }\end{array}$} & \multicolumn{4}{|c|}{ Ajuste 6 puntos } & \multicolumn{4}{|c|}{ Ajuste 4 puntos } \\
\hline & \multicolumn{2}{|c|}{ Polinómico } & \multicolumn{2}{|c|}{ Potencial } & \multicolumn{2}{|c|}{ Polinómico } & \multicolumn{2}{|c|}{ Potencial } \\
\hline & Mín & Máx & Mín & Máx & Mín & Máx & Mín & Máx \\
\hline W/Rh & 0 & 0.23 & -0.57 & -2.42 & 0.78 & 3.14 & -0.03 & 4.78 \\
\hline IMedial & \multicolumn{2}{|c|}{0.09} & \multicolumn{2}{|c|}{1.54} & \multicolumn{2}{|c|}{2.01} & \multicolumn{2}{|c|}{2.9} \\
\hline W/Ag & -0.01 & 0.13 & -0.9 & -3.85 & 0.77 & 3.62 & -0.02 & 6.49 \\
\hline IMedial & \multicolumn{2}{|c|}{0.06} & \multicolumn{2}{|c|}{2.44} & \multicolumn{2}{|c|}{2.35} & \multicolumn{2}{|c|}{3.61} \\
\hline W/Al & -0.01 & -0.29 & -0.52 & -2.02 & 0.01 & 1.11 & -0.3 & 6.93 \\
\hline IMedial & \multicolumn{2}{|c|}{0.11} & \multicolumn{2}{|c|}{1.36} & \multicolumn{2}{|c|}{0.59} & \multicolumn{2}{|c|}{2.31} \\
\hline
\end{tabular}

Tabla 1. Diferencias mínimas, máximas y medias entre los valores del kerma medidos y los calculados con los ajustes polinómico y potencial para 6 y 4 puntos respectivamente. 
Tabla 2. Dosis glandular calculada a partir de ajuste polinómico y potencial a partir de 6 y 4 valores medidos del kerma para el modo 2D.

\begin{tabular}{c|c|c|c|c} 
Ajuste & \multicolumn{2}{|c|}{ Polinómico } & \multicolumn{2}{c}{ Potencial } \\
\hline PMMA (cm) & $\begin{array}{c}\text { DG ajuste 6 puntos } \\
\text { (mGy) }\end{array}$ & $\begin{array}{c}\text { DG ajuste 4 puntos } \\
\text { (m Gy) }\end{array}$ & $\begin{array}{c}\text { DG ajuste 6 puntos } \\
\text { (m Gy) }\end{array}$ & $\begin{array}{c}\text { DG ajuste 4 puntos } \\
\text { (mGy) }\end{array}$ \\
\hline $\mathbf{2}$ & 0.75 & 0.75 & 0.80 & 0.74 \\
\hline $\mathbf{3}$ & 1.01 & 1.00 & 1.04 & 0.98 \\
\hline $\mathbf{4}$ & 1.36 & 1.32 & 1.34 & 1.30 \\
\hline $\mathbf{4 . 5}$ & 1.67 & 1.62 & 1.64 & 1.60 \\
\hline $\mathbf{5}$ & 1.84 & 1.81 & 1.81 & 1.81 \\
\hline $\mathbf{6}$ & 2.12 & 2.12 & 2.06 & 2.07 \\
\hline $\mathbf{7}$ & 2.39 & 2.39 & 2.41 & 2.53 \\
\hline
\end{tabular}
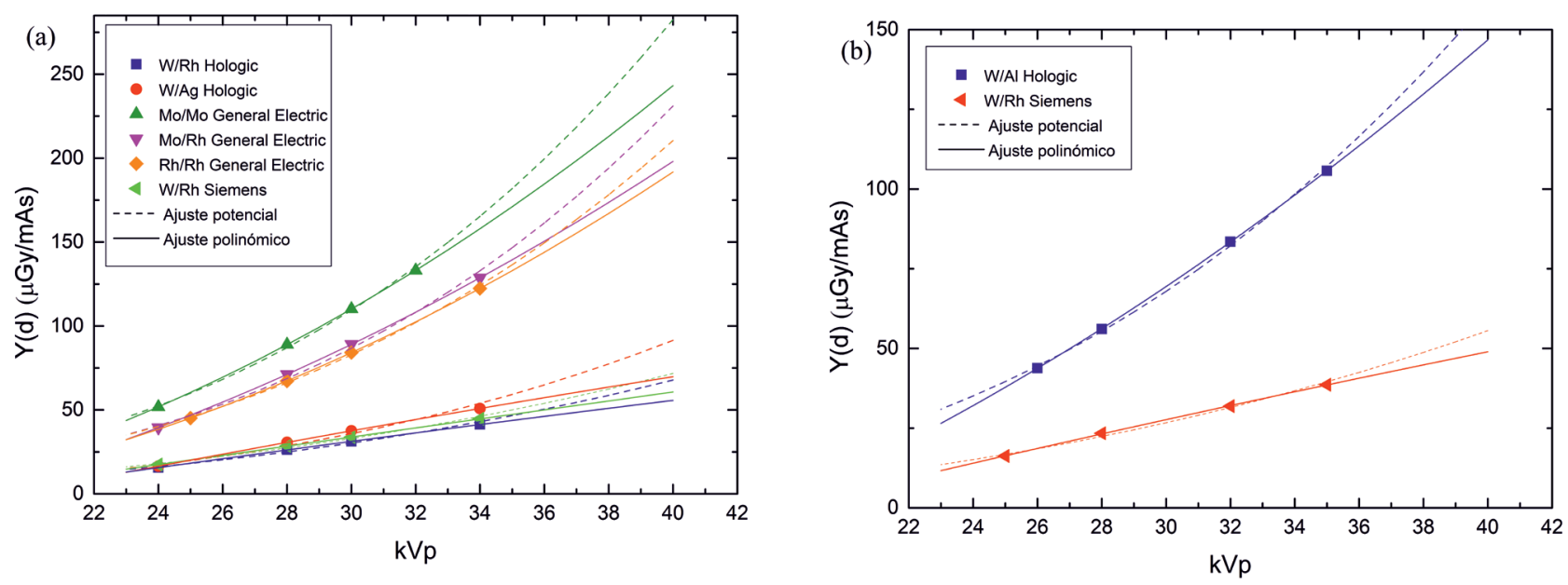

Fig. 3. Ajustes de los rendimientos medidos con cámara de ionización Nomex (PTW) a funciones polinómica y potencial. Los puntos representan los valores medidos. (a) Modo MD (b) Modo TDM.

el ajuste polinómico. En cambio, para el ajuste potencial se encuentran diferencias mucho mayores, llegando a alcanzar hasta el $6 \%$ en algunos casos.

En la Fig. 3 y Fig. 4 se muestran los valores medidos con cada detector, así como los diferentes ajustes de kerma en función del kVp. En las tablas 4 y 5 se incluyen las ecuaciones de ajuste y los coeficientes de determinación observándose que alcanzan valores más cercanos a 1 en el ajuste polinómico que en el potencial.

\section{CHR}

En la Fig. 5 se muestran las gráficas comparativas de los valores medidos y los calculados, obteniéndose unas diferencias para todos los mamógrafos y calidades de haz inferiores al $1 \%$ entre los valores medidos y los del ajuste realizado.

Se obtuvieron en todos los casos valores menores de la CHR con el multímetro que con la cámara de ionización, variando entre un 7\% hasta un 16\% inferior dependiendo de la calidad de haz.

\section{Dosis glandular}

Las Tablas 6 y 7 muestran los resultados del cálculo de la dosis glandular en todos los mamógrafos y la diferencia que existe teniendo en cuenta el kerma calculado mediante el ajuste polinómico y el potencial.

En el equipo Selenia Dimensions se encuentran unas discrepancias en torno al 3\% de media, llegando en el caso del multímetro y el modo TDM hasta el $12 \%$. 
Tabla 3a. Diferencias relativas en tanto por ciento de los valores del kerma calculados con ajuste polinómico y potencial respecto de los valores medidos, para todas las calidades de haz disponibles en cada mamógrafo. (a) Cámara de ionización (PTW). (b) NOMEX multímetro (PTW).

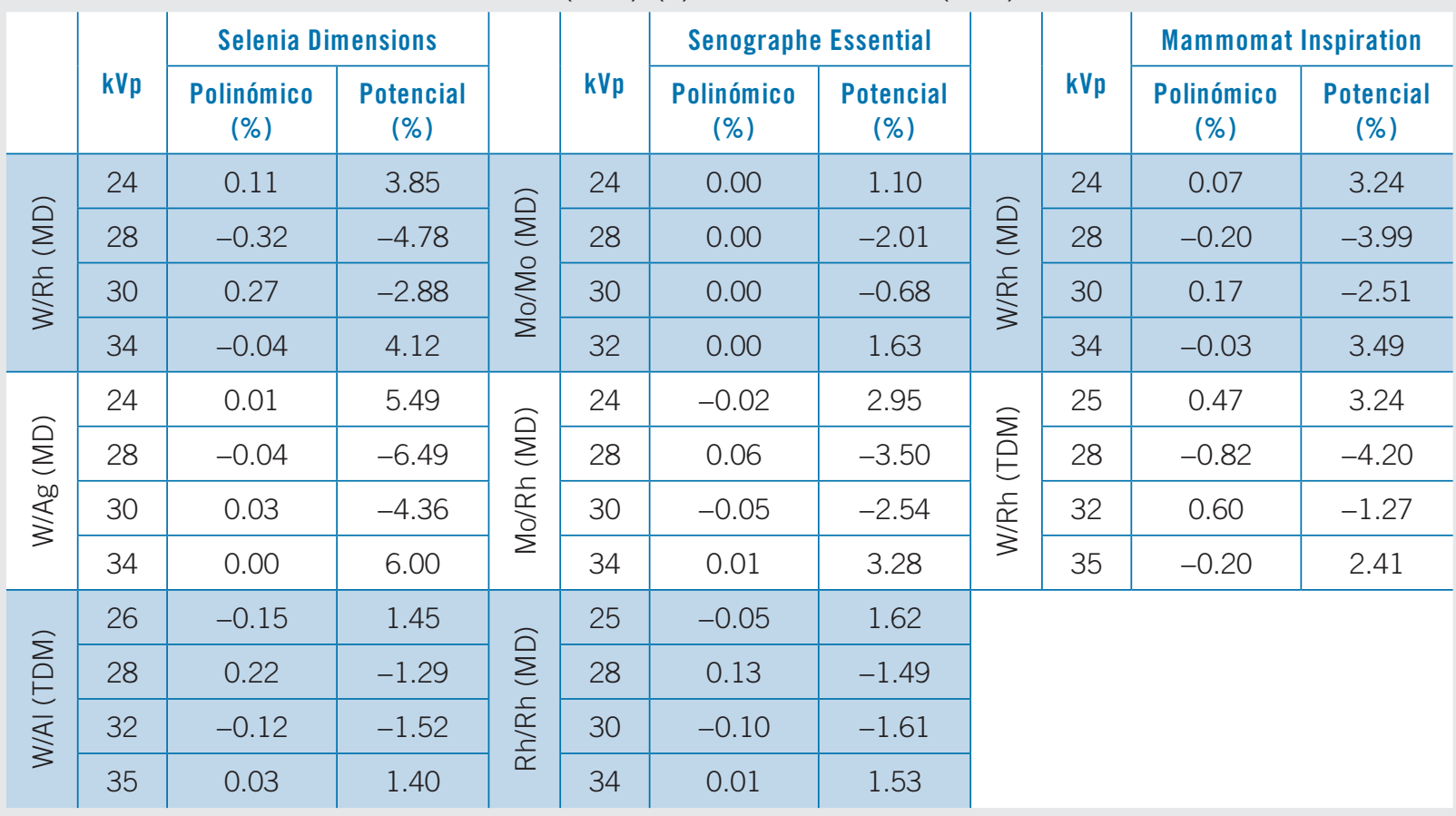

Tabla 3b.

\begin{tabular}{|c|c|c|c|c|c|c|c|c|c|c|c|}
\hline & \multirow[b]{2}{*}{ kVp } & \multicolumn{2}{|c|}{ Selenia Dimensions } & & \multirow[b]{2}{*}{$k V p$} & \multicolumn{2}{|c|}{ Senographe Essential } & & \multirow[b]{2}{*}{$k V p$} & \multicolumn{2}{|c|}{ Mammomat Inspiration } \\
\hline & & $\begin{array}{c}\text { Polinómico } \\
(\%)\end{array}$ & $\begin{array}{c}\text { Potencial } \\
(\%)\end{array}$ & & & $\begin{array}{c}\text { Polinómico } \\
(\%)\end{array}$ & $\begin{array}{c}\text { Potencial } \\
(\%)\end{array}$ & & & $\begin{array}{c}\text { Polinómico } \\
(\%)\end{array}$ & $\begin{array}{c}\text { Potencial } \\
(\%)\end{array}$ \\
\hline \multirow{4}{*}{ 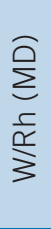 } & 24 & 0.06 & 3.96 & \multirow{4}{*}{ 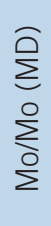 } & 24 & -0.01 & 1.19 & \multirow{4}{*}{ 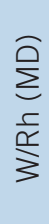 } & 24 & 0.04 & 3.35 \\
\hline & 28 & -0.17 & -4.81 & & 28 & 0.02 & -2.17 & & 28 & -0.13 & -4.07 \\
\hline & 30 & 0.14 & -3.12 & & 30 & -0.02 & -0.76 & & 30 & 0.11 & -2.68 \\
\hline & 34 & -0.02 & 4.30 & & 32 & 0.01 & 1.78 & & 34 & -0.02 & 3.65 \\
\hline \multirow{4}{*}{ 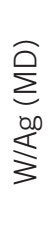 } & 24 & 0.04 & 5.43 & \multirow{4}{*}{$\begin{array}{l}\widehat{\partial} \\
\sum \\
\frac{c}{\underline{\Sigma}} \\
\frac{0}{\Sigma}\end{array}$} & 24 & -0.03 & 3.07 & \multirow{4}{*}{ 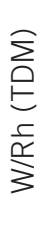 } & 25 & 0.11 & 2.62 \\
\hline & 28 & -0.11 & -6.47 & & 28 & 0.08 & -3.67 & & 28 & -0.19 & -3.22 \\
\hline & 30 & 0.09 & -4.25 & & 30 & -0.06 & -2.56 & & 32 & 0.14 & -1.54 \\
\hline & 34 & -0.01 & 5.91 & & 34 & 0.01 & 3.37 & & 35 & -0.05 & 2.27 \\
\hline \multirow{4}{*}{ 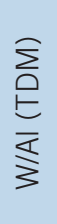 } & 26 & 0.41 & 4.26 & \multirow{4}{*}{$\begin{array}{l}\widehat{D} \\
\frac{c}{\underline{\alpha}} \\
\frac{\frac{r}{\alpha}}{\alpha}\end{array}$} & 25 & -0.03 & 1.72 & & & & \\
\hline & 28 & -0.81 & -3.93 & & 28 & 0.09 & -1.68 & & & & \\
\hline & 32 & 0.49 & -4.23 & & 30 & -0.06 & -1.55 & & & & \\
\hline & 35 & -0.06 & -0.39 & & 34 & 0.01 & 1.56 & & & & \\
\hline
\end{tabular}

En el caso de Senographe Essential, las diferencias se mantienen constantes para todas las calidades de haz, encontrándose alrededor de un $2 \%$. En el equipo Mammomat Inspiration se obtiene un resultado similar al de Hologic, siendo las diferencias medias del 3\% pero alcanzando en el caso del multímetro para el modo TDM discrepancias de hasta casi el 8\%.

Por otra parte, se muestra en la tabla 8 la diferencia obtenida en la dosis glandular determinada a partir de las medidas con la cámara de ionización y el multíme- 

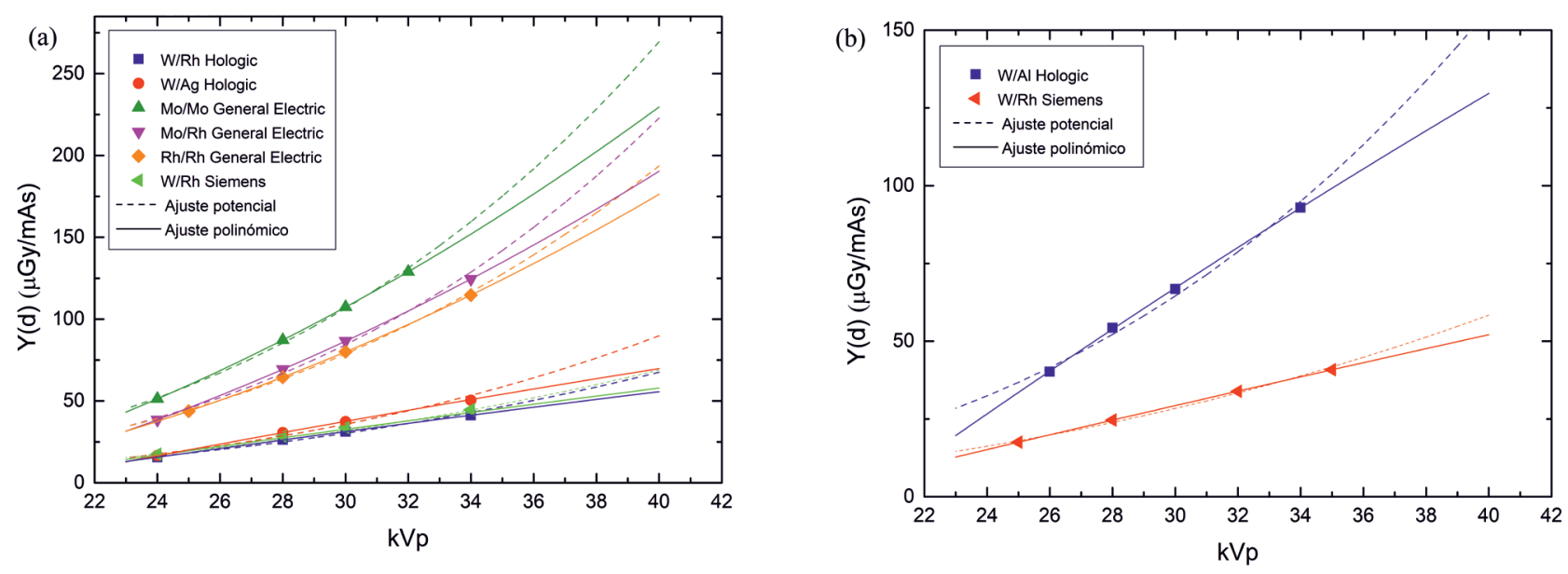

Fig. 4. Ajustes de los rendimientos medidos con Nomex Multímetro (PTW) a funciones polinómica y potencial. (a) Modo MD. (b) Modo TDM.

Tabla 4. Coeficientes obtenidos con los ajustes polinómico y potencial así como los coeficientes de determinación de cada ajuste para las medidas con cámara de ionización.

\begin{tabular}{c|c|c|c|c|c|c|c} 
& \multicolumn{4}{|c|}{$K=d+e \times k V p+f \times k V p^{2}$} & \multicolumn{3}{c}{$K=A(k V p)^{n}$} \\
\hline Modo MD & $d$ & $e$ & $f$ & $\boldsymbol{R}^{2}$ & $\boldsymbol{A}$ & $\boldsymbol{n}$ & $\boldsymbol{R}^{2}$ \\
\hline W/Rh Hologic & -54.4 & -3.17 & -0.01 & 0.9993 & 0.0022 & 2.798 & 0.9874 \\
\hline W/Ag Hologic & -80.73 & 4.48 & -0.02 & 1 & 0.0006 & 3.251 & 0.9813 \\
\hline $\begin{array}{c}\text { Mo/Mo General } \\
\text { Electric }\end{array}$ & -20.05 & -2.38 & 0.22 & 1 & 0.0015 & 3.298 & 0.9983 \\
\hline $\begin{array}{c}\text { Mo/Rh General } \\
\text { Electric }\end{array}$ & -39.86 & -0.66 & 0.16 & 1 & 0.0008 & 3.402 & 0.9948 \\
\hline $\begin{array}{c}\text { Rh/Rh General } \\
\text { Electric }\end{array}$ & -1.35 & -3.09 & 0.20 & 0.9999 & 0.0013 & 3.241 & 0.9981 \\
\hline $\begin{array}{c}\text { W/Rh Siemens } \\
\text { Modo TDM }\end{array}$ & -50.47 & -2.91 & 0.01 & 0.9999 & 0.0033 & 2.705 & 0.9904 \\
\hline W/Al Hologic & -49.64 & 1.47 & 0.09 & 0.9999 & 0.0029 & 2.960 & 0.9983 \\
\hline W/Rh Siemens & -47.28 & 2.77 & -0.01 & 0.9991 & 0.0046 & 2.548 & 0.9916
\end{tabular}

tro. La discrepancia resulta mayor para el modo TDM que para el modo DM, y de forma general se obtienen diferencias ligeramente menores con el ajuste polinómico que con el potencial.

\section{Discusión y conclusiones}

Se ha analizado la relación del kerma con el kVp para tres equipos de mamografía diferentes, utilizando un detector semiconductor y una cámara de ionización, mediante el ajuste a 6 y 4 valores de medida. Se han comparado los resultados obtenidos con el ajuste a una función potencial y con el ajuste a una función polinómica de segundo grado. Los resultados son esclarecedores en cuanto a que, como no podía ser de otra manera, los ajustes utilizando 6 puntos siempre resultan mejores que usando 4 valores, pero la repercusión que tiene utilizar menos medidas para el kerma en la dosis glandular es mínima.

Los resultados obtenidos muestran, además, que el ajuste del kerma (kVp) a un polinomio de segundo grado es mejor que utilizar el ajuste potencial. Puesto que a tensión cero la dosis es cero, inicialmente se ajustó el valor del rendimiento a una función polinómica sin término independiente del tipo: 

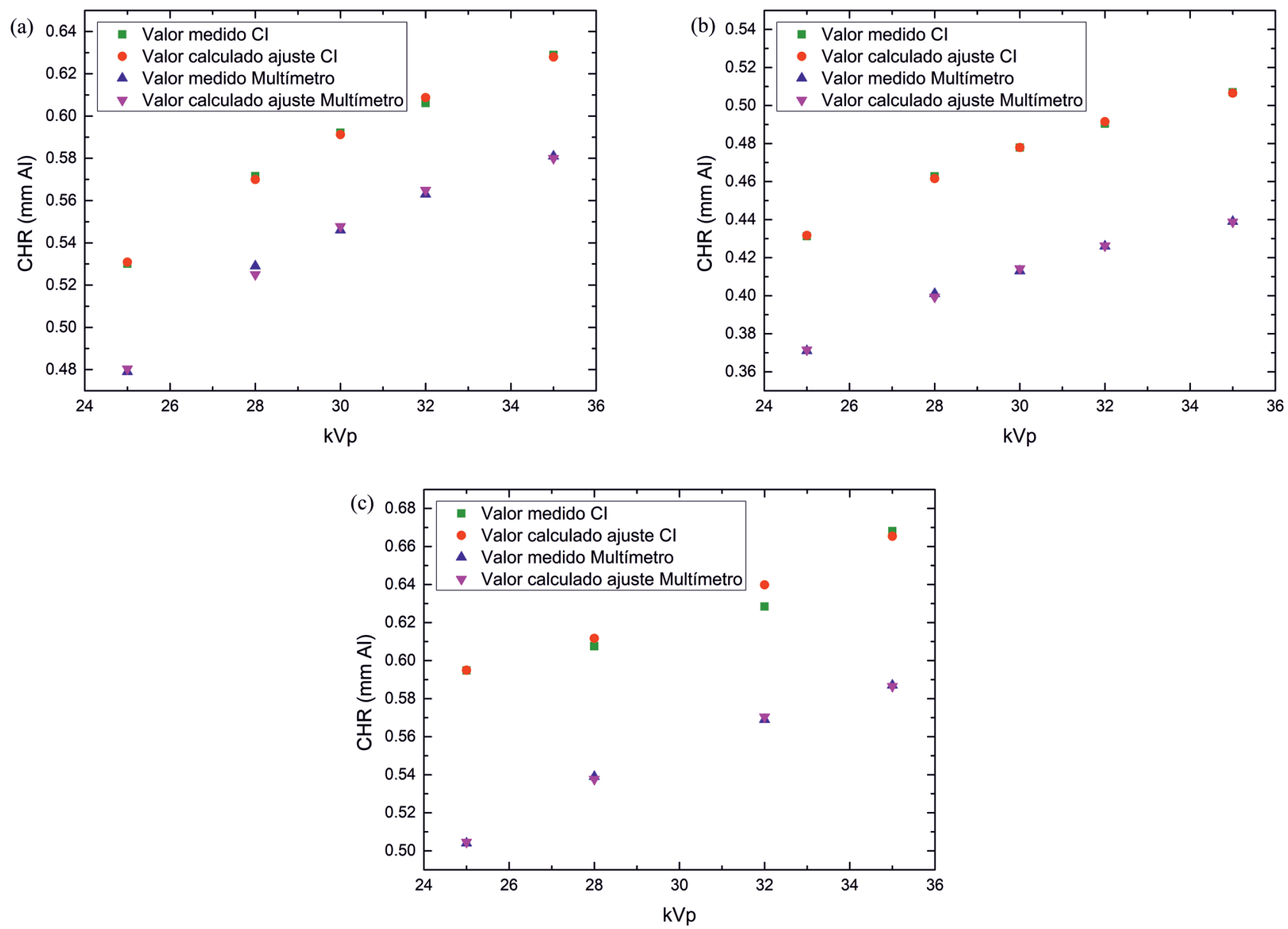

Fig. 5. Capas hemirreductoras medidas con cámara de ionización y multímetro, y calculadas mediante ajuste polinómico para diferentes mamógrafos y calidad de haz. (a) W/Rh (2D) en Hologic Selenia Dimensions. (b) Mo/Rh (2D) en Senographe Essential de General Electric. (c) W/Rh (3D) en Siemens Mammomat Inspiration.

Tabla 5. Coeficientes obtenidos con los ajustes polinómico y potencial así como los coeficientes de determinación de cada ajuste para las medidas con multímetro.

\begin{tabular}{|c|c|c|c|c|c|c|c|}
\hline \multirow[b]{2}{*}{ Modo MD } & \multicolumn{4}{|c|}{$K=d+e \times k V p+f \times k V p^{2}$} & \multicolumn{3}{|c|}{$K=A(k V p)^{n}$} \\
\hline & $d$ & $e$ & $f$ & $R^{2}$ & $A$ & $n$ & $R^{2}$ \\
\hline W/Rh Hologic & -57.46 & 3.39 & -0.01 & 0.9999 & 0.0023 & 2.792 & 0.9865 \\
\hline W/Ag Hologic & -81.67 & 4.6 & -0.02 & 1 & 0.0007 & 3.197 & 0.9811 \\
\hline $\begin{array}{c}\text { Mo/Mo General } \\
\text { Electric }\end{array}$ & -43.15 & -0.39 & 0.18 & 1 & 0.0018 & 3.224 & 0.9979 \\
\hline $\begin{array}{c}\text { Mo/Rh General } \\
\text { Electric }\end{array}$ & -46.91 & 0.001 & 0.15 & 1 & 0.0019 & 3.377 & 0.9944 \\
\hline $\begin{array}{c}\text { Rh/Rh General } \\
\text { Electric }\end{array}$ & -18.13 & -1.49 & 0.16 & 1 & 0.0019 & 3.126 & 0.9979 \\
\hline W/Rh Siemens & -51.87 & -3.05 & -0.01 & 1 & 0.0034 & 2.690 & 0.9895 \\
\hline \multicolumn{8}{|l|}{ Modo TDM } \\
\hline W/Al Hologic & -157.70 & 8.42 & -0.03 & 0.9998 & 0.0018 & 3.082 & 0.9924 \\
\hline W/Rh Siemens & -44.95 & 2.62 & -0.01 & 1 & 0.0056 & 2.506 & 0.9940 \\
\hline
\end{tabular}


Tabla 6a. Dosis glandular calculada a partir de ajuste polinómico y potencial del rendimiento para medidas con la cámara de ionización en los diferentes equipos. (a) Modo MD. (b) Modo TDM.

\begin{tabular}{c|c|c|c|c|c|c|c|c|c}
\multirow{2}{*}{$\begin{array}{c}\text { PMMA } \\
(\mathbf{c m})\end{array}$} & \multicolumn{3}{|c|}{ Selenia Dimensions } & \multicolumn{3}{c|}{ Senographe Essential } & \multicolumn{3}{c}{ Mammomat Inspiration } \\
\cline { 2 - 10 } & $\begin{array}{c}\text { DG (mGy) } \\
\text { ajuste } \\
\text { polinómico }\end{array}$ & $\begin{array}{c}\text { DG (mGy) } \\
\text { ajuste } \\
\text { potencial }\end{array}$ & $\begin{array}{c}\text { Diferencia } \\
(\%)\end{array}$ & $\begin{array}{c}\text { DG (mGy) } \\
\text { ajuste } \\
\text { polinómico }\end{array}$ & $\begin{array}{c}\text { DG (mGy) } \\
\text { ajuste } \\
\text { potencial }\end{array}$ & $\begin{array}{c}\text { Diferencia } \\
(\%)\end{array}$ & $\begin{array}{c}\text { DG (mGy) } \\
\text { ajuste } \\
\text { polinómico }\end{array}$ & $\begin{array}{c}\text { DG (mGy) } \\
\text { ajuste } \\
\text { potencial }\end{array}$ & $\begin{array}{c}\text { Diferencia } \\
(\%)\end{array}$ \\
\hline 2 & 0.75 & 0.74 & 2.2 & 0.89 & 0.87 & 1.8 & 0.45 & 0.44 & 2.5 \\
\hline 3 & 1.00 & 0.98 & 2.2 & 0.94 & 0.92 & 2.4 & 0.60 & 0.58 & 3.5 \\
\hline 4 & 1.32 & 1.30 & 1.7 & 1.06 & 1.05 & 1.7 & 0.84 & 0.80 & 3.8 \\
\hline 4.5 & 1.62 & 1.60 & 1.2 & 1.36 & 1.34 & 1.7 & - & - & - \\
\hline 5 & 1.81 & 1.81 & 0.1 & 1.33 & 1.31 & 1.7 & 1.14 & 1.11 & 2.7 \\
\hline 6 & 2.12 & 2.07 & 2.4 & 2.03 & 2.00 & 1.7 & 1.60 & 1.58 & 1.5 \\
\hline 7 & 2.39 & 2.53 & 5.7 & 2.58 & 2.55 & 1.0 & - & - & - \\
\hline
\end{tabular}

Tabla 6b.

\begin{tabular}{c|c|c|c|c|c|c}
\multirow{2}{*}{$\begin{array}{c}\text { PMMA } \\
(\mathbf{c m})\end{array}$} & \multicolumn{3}{|c|}{ Selenia Dimensions } & \multicolumn{3}{c}{ Mammomat Inspiration } \\
\cline { 2 - 7 } & $\begin{array}{c}\text { DG (mGy) } \\
\text { ajuste } \\
\text { polinómico }\end{array}$ & $\begin{array}{c}\text { DG (mGy) } \\
\text { ajuste } \\
\text { potencial }\end{array}$ & $\begin{array}{c}\text { Diferencia } \\
(\%)\end{array}$ & $\begin{array}{c}\text { DG (mGy) } \\
\text { ajuste } \\
\text { polinómico }\end{array}$ & $\begin{array}{c}\text { DG (mGy) } \\
\text { ajuste } \\
\text { potencial }\end{array}$ & $\begin{array}{c}\text { Diferencia } \\
(\%)\end{array}$ \\
\hline 2 & 1.21 & 1.23 & 1.6 & 0.71 & 0.71 & 0.5 \\
\hline 3 & 1.27 & 1.25 & 1.5 & 0.94 & 0.91 & 3.1 \\
\hline 4 & 1.66 & 1.62 & 2.2 & 1.38 & 1.30 & 5.3 \\
\hline 4.5 & 2.06 & 2.02 & 1.9 & 1.59 & 1.48 & 6.4 \\
\hline 5 & 2.51 & 2.49 & 0.7 & 1.83 & 1.69 & 7.4 \\
\hline 6 & 3.77 & 3.87 & 2.5 & 2.47 & 2.27 & 7.9 \\
\hline 7 & 4.87 & 5.44 & 10.5 & 3.12 & 3.07 & 1.9 \\
\hline
\end{tabular}

$$
K_{k V p}=e \times k V p+f \times k V p^{2}
$$

Los resultados fueron claramente favorables a utilizar este ajuste polinómico en lugar de un ajuste potencial, obteniéndose una diferencia entre los valores del kerma en aire medidos y calculados inferior para el ajuste polinómico en todos los casos.

Respecto a la dosis glandular, los resultados fueron en general mejores para el ajuste polinómico. Sin embargo, en el caso de una función polinómica de segundo grado completa, los resultados fueron aún mejores. Por tanto, ya que lo que se busca es el mejor ajuste posible para minimizar las incertidumbres en el cálculo de la dosis glandular, se utilizó finalmente una ecuación polinómica con tres grados de libertad, ya que ajusta mucho mejor los puntos de medida con los calculados.

Por tanto, los valores conseguidos mediante el ajuste polinómico de segundo grado resultan siempre más precisos que los valores obtenidos mediante el ajuste potencial. Hay que tener en cuenta, además, que los kilovoltajes utilizados en mamografía están comprendidos en un rango limitado y muy próximos entre ellos, por lo que es factible ajustar a una función polinómica.

La estimación de la dosis glandular utilizando el detector de referencia (cámara de ionización) a partir del kerma obtenido mediante la función potencial de ajuste y la polinómica, muestran diferencias inferiores a un $2.5 \%$ en todos los equipos excepto para espesores de mama grandes (mayores de $6 \mathrm{~cm}$ ). 
Tabla 7a. Dosis glandular calculada a partir de ajuste polinómico y potencial del rendimiento para medidas con el multímetro en los diferentes equipos. (a) Modo MD. (b) Modo TDM.

\begin{tabular}{|c|c|c|c|c|c|c|c|c|c|}
\hline \multirow[b]{2}{*}{$\begin{array}{l}\text { PMMA } \\
\text { (cm) }\end{array}$} & \multicolumn{3}{|c|}{ Selenia Dimensions } & \multicolumn{3}{|c|}{ Senographe Essential } & \multicolumn{3}{|c|}{ Mammomat Inspiration } \\
\hline & $\begin{array}{l}\text { DG (mGy) } \\
\text { ajuste } \\
\text { polinómico }\end{array}$ & $\begin{array}{l}\text { DG (mGy) } \\
\text { ajuste } \\
\text { potencial }\end{array}$ & $\begin{array}{c}\text { Diferencia } \\
(\%)\end{array}$ & $\begin{array}{l}\text { DG (mGy) } \\
\text { ajuste } \\
\text { polinómico }\end{array}$ & $\begin{array}{l}\text { DG (mGy) } \\
\text { ajuste } \\
\text { potencial }\end{array}$ & $\begin{array}{c}\text { Diferencia } \\
(\%)\end{array}$ & $\begin{array}{l}\text { DG (mGy) } \\
\text { ajuste } \\
\text { polinómico }\end{array}$ & $\begin{array}{l}\text { DG (mGy) } \\
\text { ajuste } \\
\text { potencial }\end{array}$ & $\begin{array}{c}\text { Diferencia } \\
(\%)\end{array}$ \\
\hline 2 & 0.71 & 0.69 & 2.1 & 0.81 & 0.80 & 2.0 & 0.40 & 0.39 & 2.6 \\
\hline 3 & 0.95 & 0.90 & 4.7 & 0.76 & 0.75 & 2.5 & 0.53 & 0.51 & 3.7 \\
\hline 4 & 1.28 & 1.20 & 6.1 & 0.94 & 0.92 & 1.8 & 0.72 & 0.70 & 3.9 \\
\hline 4.5 & 1.58 & 1.49 & 5.7 & 1.20 & 1.18 & 1.8 & - & - & - \\
\hline 5 & 1.73 & 1.67 & 3.4 & 1.17 & 1.15 & 1.8 & 0.99 & 0.96 & 2.8 \\
\hline 6 & 1.89 & 1.82 & 4.0 & 1.79 & 1.76 & 1.8 & 1.38 & 1.36 & 1.6 \\
\hline 7 & 2.13 & 2.22 & 4.0 & 2.28 & 2.26 & 1.1 & - & - & - \\
\hline
\end{tabular}

Tabla $7 \mathrm{~b}$.

\begin{tabular}{c|c|c|c|c|c|c}
\multirow{2}{*}{$\begin{array}{c}\text { PMMA } \\
(\mathbf{c m})\end{array}$} & \multicolumn{3}{|c|}{ Selenia Dimensions } & \multicolumn{3}{c}{ Mammomat Inspiration } \\
\cline { 2 - 7 } & $\begin{array}{c}\text { DG (mGy) } \\
\text { ajuste } \\
\text { polinómico }\end{array}$ & $\begin{array}{c}\text { DG (mGy) } \\
\text { ajuste } \\
\text { potencial }\end{array}$ & $\begin{array}{c}\text { Diferencia } \\
(\%)\end{array}$ & $\begin{array}{c}\text { DG (mGy) } \\
\text { ajuste } \\
\text { polinómico }\end{array}$ & $\begin{array}{c}\text { DG (mGy) } \\
\text { ajuste } \\
\text { potencial }\end{array}$ & $\begin{array}{c}\text { Diferencia } \\
(\%)\end{array}$ \\
\hline 2 & 1.00 & 0.98 & 2.0 & 0.70 & 0.70 & 0.4 \\
\hline 3 & 1.01 & 0.99 & 2.0 & 1.01 & 0.98 & 3.0 \\
\hline 4 & 1.30 & 1.29 & 1.0 & 1.58 & 1.53 & 3.1 \\
\hline 4.5 & 1.64 & 1.63 & 0.3 & 1.97 & 1.94 & 1.7 \\
\hline 5 & 2.04 & 2.08 & 1.6 & 2.36 & 2.38 & 0.8 \\
\hline 6 & 3.27 & 3.44 & 4.9 & 3.33 & 3.46 & 3.9 \\
\hline 7 & 4.67 & 5.32 & 12.2 & 4.31 & 4.65 & 7.2 \\
\hline
\end{tabular}

Se ha comprobado un mejor ajuste para el kerma $(\mathrm{k} V \mathrm{p})$ con la función polinómica propuesta, pero al margen de la mejor adecuación en sí de este tipo de función a la respuesta de los equipos, también existe una explicación meramente matemática.

Si los datos de los que se dispone cumplen una relación de tipo exponencial de la forma siguiente:

$$
y=A x^{n}
$$

O en su defecto, se sospecha que la puedan cumplir, el ajuste habitualmente utilizado para aproximar estos datos a la función es el método de los mínimos cuadrados. Este método consiste en minimizar el error cuadrático cometido con la ayuda de dos parámetros arbitrarios, A y $n$. La expresión del error cuadrático para una función de este tipo es:

$$
E=\sum_{i=1}^{m}\left(y_{i}-A x_{i}^{n}\right)^{2}
$$

Al minimizar esta ecuación se obtiene un sistema de ecuaciones no resoluble por métodos analíticos, cuya única resolución es mediante métodos numéricos. Para solventar este problema, el procedimiento más comúnmente utilizado consiste en considerar el logaritmo de la ecuación a la que queremos ajustar los datos, obteniendo de esta manera una relación lineal:

$$
\ln y=\ln n+A \ln x
$$


Tabla 8. Diferencia en tanto por ciento en los valores de la dosis glandular con cámara de ionización y multímetro para el ajuste del kerma polinómico y potencial.

\begin{tabular}{c|c|c|c}
\multirow{2}{*}{ Mamógrafo } & \multirow{2}{*}{ Modo } & \multicolumn{2}{|c}{ Diferencia entre cámara y multímetro (\%) } \\
\cline { 2 - 4 } & & Polinómico & Potencial \\
\hline \multirow{2}{*}{ Selenia Dimensions } & MD & 6.2 & 8.7 \\
\cline { 2 - 4 } & TDM & 16.6 & 15.9 \\
\hline \multirow{2}{*}{ Senographe Essential } & MD & 12.4 & 12.5 \\
\hline \multirow{2}{*}{ Mammomat Inspiration } & MD & 12.7 & 12.8 \\
\cline { 2 - 4 } & TDM & 16.7 & 20.6
\end{tabular}

Sin embargo, la solución obtenida de esta forma no es la que se hubiese logrado con el método de los mínimos cuadrados para el problema original, y esta aproximación puede, en algunos casos, diferir significativamente del resultado conseguido si no se hubiese utilizado el logaritmo para transformar la ecuación. ${ }^{23}$

Por lo tanto, el ajuste polinómico se corresponde mejor con el problema original que el ajuste potencial utilizando la aproximación logarítmica para resolver el sistema de ecuaciones. Esto coincide con los resultados obtenidos en este trabajo.

Por tanto, se propone utilizar el ajuste a una función polinómica para la función de kerma $(k V p)$ en lugar de la potencial que propone el Protocolo Español de Control de Calidad en Radiodiagnóstico.

Por otra parte, las diferencias encontradas en la estimación de la dosis glandular a partir de las medidas realizadas con la cámara de ionización y con el detector semiconductor se encuentran alrededor del $10 \%$, por lo que el cálculo de la dosis glandular es muy dependiente del detector utilizado para la obtención del kerma y la CHR. Esto implica que el detector tiene una mayor influencia porcentual en la estimación de la dosis glandular que la influencia por el tipo de función de ajuste empleado para la obtención del kerma. Por esta razón, es fundamental conocer la respuesta de los diferentes detectores disponibles, pues pequeñas variaciones en las medidas afecta en un porcentaje considerable a la estimación de la dosis glandular.

Como conclusión, es importante utilizar el mismo equipo de medida en los controles de calidad, con la misma disposición y con una adecuada metodología de ajuste que minimice las incertidumbres obtenidas en la estimación de la dosis glandular.

En todo caso, es imprescindible tener bien caracterizados nuestros detectores y conocer cómo afectan a la dosis glandular.

\section{Bibliografía}

1. Yaffe MJ, Mainprize JG. Risk of Radiation-induced Breast Cancer from Mammographic Screening. Radiology 2011; 258(1):98-105.

2. International Comission on Radiation Units and Measurements. Conversion coefficients for use in radiological protection against external radiation. ICRU report 74: Oxford University Press;2005.

3. International Comission on Radiological Protection. Statement from the 1987 Como Meeting of the ICRP. Publication 52: Pergamon Press; 1987.

4. Dance DR. Monte Carlo calculation of conversion factors for the estimation of mean glandular breast dose. Phys Med Biol. 1990;35(9):1211-9.

5. Dance DR, Skinner CL, Young KC, Beckett JR, Kotre CJ. Additional factors for the estimation of mean glandular breast dose using the UK mammography dosimetry protocol. Phys Med Biol. 2000;45(11):3225-40.

6. Dance DR, Young KC, Van Engen RE. Further factors for the estimation of mean glandular dose using the United Kingdom, European and IAEA breast dosimetry protocols. Phys Med Biol. 2009; 54(14): 4361-4372.

7. Sobol WT, Wu X. Parametrization of mammography normalized average glandular dose tables. Med Phys. 1997;24(4): 547-54.

8. Boone JM. Normalized glandular dose (DgN) coefficients for arbitrary $\mathrm{x}$-ray spectra in mammography: computer-fit values of Monte Carlo derived data. Med Phys. 2002;29(5): 869-75.

9. Castillo M, Garayoa J, Estrada C, Tejerina A, Benítez O, Alcazar A, et al. Tomosíntesis de mama: mamografía sintetizada versus mamografía digital. Impacto en la dosis. Rev Senol Patol Mamar. 2015; 28(1): 3-10.

10. Zuley M, Bandos A, Ganott M, Sumkin J, Kelly A, Catullo V, et al. Digital Breast Tomosynthesis versus supplemental diagnostic mammographic views for evaluation of noncalcified breast lesions. Radiology. 2013;266(1):89-95.

11. Skaane $P$, Bandos A, Eben E, Jebsen I, Krager M, Haakenaasen $U$, et al. Two-view digital breast tomosynthesis screening with synthetically reconstructed projection images: comparison with digital breast tomosynthesis with full-field digital mammographic images. Radiology. 2014;271:65563.

12. Skaane P, Bandos A, Gulien R, Eben E, Ekseth U, Haakenaasen $U$ et al. Comparison of digital mammography 
alone and digital mammography plus tomosynthesis in a population-based screening program. Radiology. 2013;267: 47-56.

13. Dance DR, Young KC, Van Engen RE. Estimation of mean glandular dose for breast tomosynthesis: Factors for use with the UK, European and IAEA breast dosimetry protocols. Phys Med Biol. 2011;56: 453-71.

14. Sechopoulos I, Sabol JM, Berglund J, Bolch WE, Brateman $\mathrm{L}$, Christodoulou $\mathrm{E}$ et al. Radiation dosimetry in digital breast tomosynthesis: Report of AAPM Tomosynthesis Subcommittee Task Group 223. Med Phys. 2014; 41(9):091501.

15. Sechopoulos I, Suryanarayanan S, Vedantham SC, Karellas A. Computation of the glandular radiation dose in digital tomosynthesis of the breast. Med Phys. 2007;34(1): 221-32.

16. Gennaro G, Avramova-Cholakova S, Azzalini A, Chapel ML, Chevalier M, Ciraj O, et al. Quality Controls in Digital Mammography protocol of the EFOMP MammoWorking group. Phys Medica. 2018;48:55-64.

17. S. Avramova-Cholakova S, Vassileva J, Borisova R, Atanasova I. An estimate of the influence of the measurement procedu- re on patient and phantom doses in breast imaging. Radiat Prot Dosimetry. 2008;129(1-3):150-4.

18. Robson K J. A parametric method for determining mammographic X-ray tube output and half value layer. $\mathrm{Br} \mathrm{J}$ Radiol 74. 2001;335-40.

19. Nickoloff EL, Berman HL. Factors affecting X-ray Spectra. Radiographics. 1993;13(6):1337-48.

20. EFOMP Mammo Working Group. Quality Controls in Digital Mammography. 2015.

21. SEFM, SEPR, SERAM. Protocolo Español de Control de Calidad en Radiodiagnóstico. Revisión 2011. Madrid: Senda Editorial; 2012.

22. R.E. Van Engen RE, Bosmans H, Bouwman RW, Dance DR, Heid P, Lazzari B, et al. Protocol for the Quality Control of the Physical and Technical Aspects of Digital Breast Tomosynthesis Systems. Version 1.03. Nijmegen; 2018.

23. Burden RL, Faires DJ, Burden AM. Approximation theory. Numerical Analysis. 10th edition. Boston: Cengage Learning; 2016. 
VersaHD.

Powered by hig

dynamic ract

Versa $\mathrm{HD}^{\mathrm{m}}$ with $\mathrm{Monaco}^{\circledR}$ gives you the clinical

flexibility of high definition dynamic radiosurgery

(HDRS) and conventional RT in a single platform.

HDRS means you can deliver stereotactic treatments

within standard RT time slots, regardless of anatomy

or complexity. And, with advanced image guidance

tools and up to $5 x$ less transmission to non-targeted

regions, you have assurance of end-to-end precision.

Discover how Versa HD can help you meet operational efficiencies while achieving better outcomes for patients.

\section{elekta.com/VersaHD}

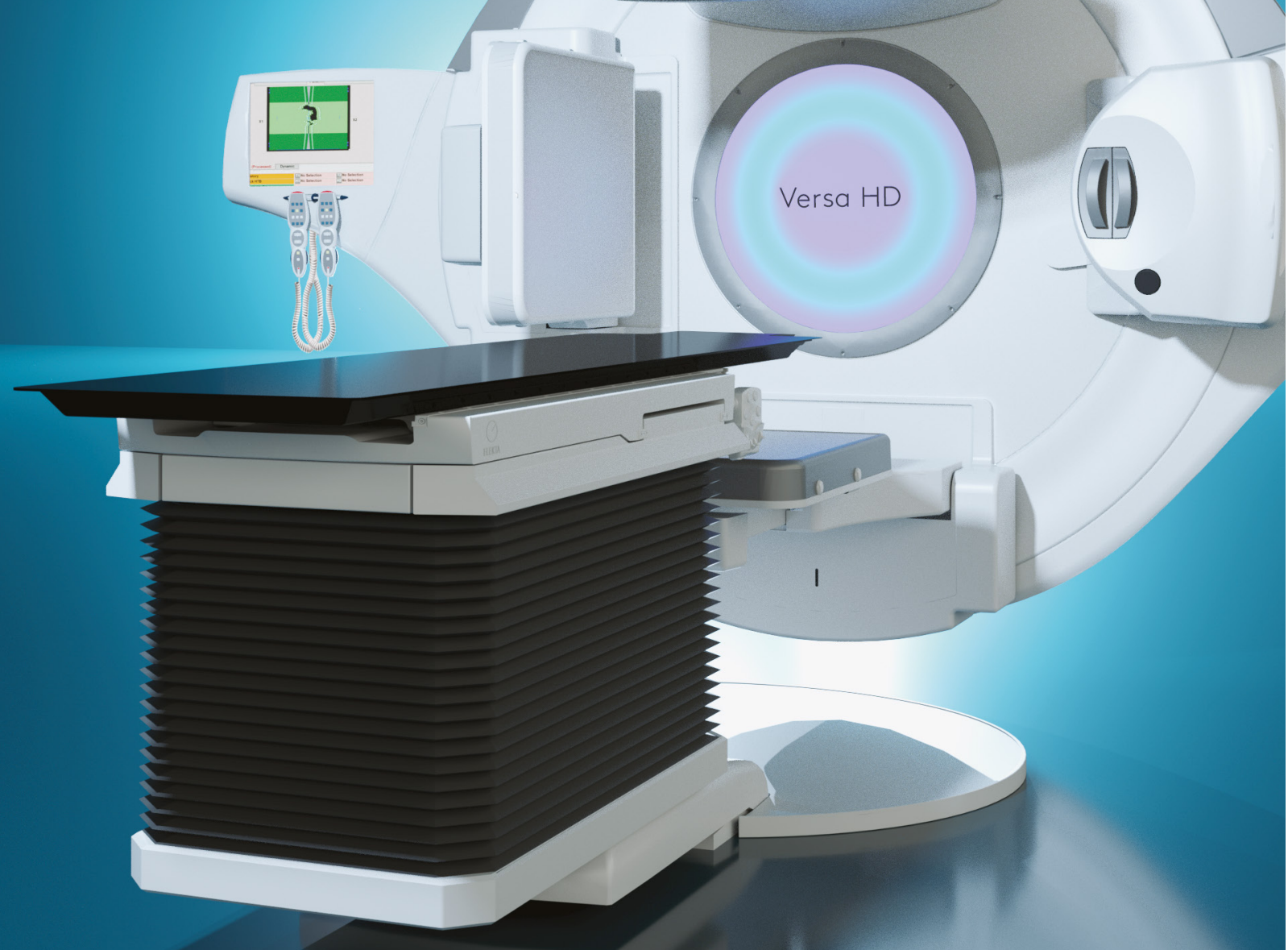

